\title{
Early neuromuscular blockade in moderate to severe acute respiratory distress syndrome: do not throw the baby out with the bathwater!
}

\author{
Sunghoon Park ${ }^{1}$, Matthieu Schmidt ${ }^{2,3}$ \\ ${ }^{1}$ Department of Pulmonary, Allergy and Critical Care Medicine, Hallym University Sacred Heart Hospital, Anyang, South Korea; ${ }^{2}$ Sorbonne \\ Université, INSERM UMRS_1166-iCAN, Institute of Cardiometabolism and Nutrition, 75651 Paris Cedex 13, France; ${ }^{3}$ Assistance Publique- \\ Hôpitaux de Paris, Pitié-Salpêtrière Hospital, Medical Intensive Care Unit, 75651 Paris Cedex 13, France \\ Correspondence to: Sunghoon Park. Department of Pulmonary, Allergy and Critical Care Medicine, Hallym University Sacred Heart Hospital, \\ 22 Gwanpyeong-ro, Anyang 14068, South Korea. Email: f2000tj@gmail.com. \\ Provenance: This is an invited article commissioned by the Section Editor Xue-Zhong Xing [National Cancer Center (NCC)/Cancer Hospital, \\ Chinese Academy of Medical Sciences (CAMS) and Peking Union Medical College (PUMC), Beijing, China]. \\ Comment on: National Heart, Lung, and Blood Institute PETAL Clinical Trials Network, Moss M, Huang DT, et al. Early Neuromuscular Blockade \\ in the Acute Respiratory Distress Syndrome. N Engl J Med 2019;380:1997-2008.
}

Submitted Sep 07, 2019. Accepted for publication Sep 26, 2019.

doi: $10.21037 /$ jtd.2019.10.25

View this article at: http://dx.doi.org/10.21037/jtd.2019.10.25

Light sedation with daily interruption and analgesiabased sedation are currently recommended in critically ill, mechanically ventilated patients (1). However, in the context of acute respiratory distress syndrome (ARDS), the benefits of this sedative strategy remain controversial. A lung protective ventilation strategy to minimize ventilatorinduced lung injury (VILI) significantly decreases mortality in patients with ARDS (2), and therefore is mandatory for early management of these patients. However, deep sedation could be required to obtain patient-ventilator synchronization (3). In particular, deep sedation involving neuromuscular blocking agents (NMBAs) is frequently needed during several adjunctive therapies, such as with high positive end-expiratory pressure (PEEP) levels, prone positioning, or extracorporeal membrane oxygenation.

The ARDS et Curarisation Systematique (ACURASYS) trial (4) was the first multicenter randomized placebocontrolled trial to test the impact of an NMBA during the early course of severe ARDS on 90-day mortality. The ACURASYS strategy combined optimization of mechanical ventilation before randomization, a moderate PEEP according to the Acute Lung Injury and Acute Respiratory Distress Syndrome (ARMA) protocol (2), recommendations of early prone positioning, and an early switch to pressure support ventilation after improvement.
This strategy, including 48 hours of cisatracurium, was significantly associated with more ventilator-free days and a lower 90-day mortality (5). Interestingly, barotrauma, defined as a newly developed pneumothorax, pneumomediastinum, subcutaneous emphysema, or pneumatocele $>2 \mathrm{~cm}$ in diameter, was more frequent in the control group. By contrast, the incidence of intensive care unit-acquired weakness was not different between the cisatracurium and control groups.

A decade later, the Reevaluation of Systematic Early Neuromuscular Blockade (ROSE) trial (5) reevaluated the beneficial effects of cisatracurium in patients with moderate to severe ARDS. This randomized controlled trial reported different results than the ACURASYS trial. Notably, although mortality was similar in the control groups in both trials, no significant difference in 90-day mortality was detected between the cisatracurium and the control groups in the ROSE trial (5). In addition, the rate of barotrauma was similar between the two groups, with more adverse cardiovascular events in the cisatracurium group.

The difference in the results between these two trials can be explained by differences in the trial protocols. First, the sample sizes were different, with 1,088 patients included in the ROSE trial ( $v s .340$ patients in the ACURASYS trial). Second, the control group was supposed to receive light 
Table 1 Comparisons of mechanical ventilation parameters (day 1) and adjunctive therapies between the ACURASYS and ROSE trials

\begin{tabular}{|c|c|c|c|c|}
\hline Variables & \multicolumn{2}{|c|}{ The ACURASYS trial } & \multicolumn{2}{|c|}{ The ROSE trial } \\
\hline Levels of sedation & $\begin{array}{l}\text { Not reported (target: } \\
\text { Ramsay score of 6) }\end{array}$ & $\begin{array}{l}\text { Not reported (target: } \\
\text { Ramsay score of } 6 \text { ) }\end{array}$ & $\begin{array}{l}\text { RASS: }-4.8 \pm 0.8 \\
\text { RSAS: } 1.1 \pm 0.4\end{array}$ & $\begin{array}{l}\text { RASS: }-2.7 \pm 1.9 \\
\text { RSAS: } 2.4 \pm 2.3\end{array}$ \\
\hline Tidal volume, $\mathrm{mL} / \mathrm{kg}$ & $6.3 \pm 0.8$ & $6.3 \pm 0.8$ & $5.9 \pm 0.8$ & $5.9 \pm 0.7$ \\
\hline Set PEEP, $\mathrm{cmH}_{2} \mathrm{O}$ & $9.6 \pm 2.7$ & $10.2 \pm 2.2$ & $12.5 \pm 4.4$ & $13.4 \pm 4.2$ \\
\hline Plateau pressure, $\mathrm{cmH}_{2} \mathrm{O}$ & $24 \pm 5$ & $23 \pm 5$ & $25.2 \pm 5.2$ & $25.6 \pm 5.6$ \\
\hline $\mathrm{pH}$ & $7.35 \pm 0.10$ & $7.35 \pm 0.08$ & $7.32 \pm 0.10$ & $7.33 \pm 0.10$ \\
\hline Corticosteroid, n (\%) & 28/177 (16\%) & $37 / 162(23 \%)$ & $109 / 482(23 \%)$ & $135 / 488(28 \%)$ \\
\hline 90-day mortality, n (\%) & $56 / 177(31.6 \%)$ & $66 / 162(40.7 \%)$ & $213(42.5 \%)$ & $216(42.8 \%)$ \\
\hline Barotrauma, n (\%) & $9(5.1 \%)$ & $19(11.7 \%)$ & $20(4.0)$ & $32(6.3 \%)$ \\
\hline ICUAW (MRC scale) & $55[46-60]$ & $55[39-60]$ & $45.7 \pm 13.9$ & $49.8 \pm 10.6$ \\
\hline
\end{tabular}

ACURASYS, ARDS et Curarisation Systematique; ROSE, Reevaluation of Systematic Early Neuromuscular Blockade; ICUAW, ICUacquired weakness; MRC, Medical Research Council scale; RASS, Richmond Agitation Sedation Score; RSAS, Riker Sedation Agitation Scale; PEEP, positive end-expiratory pressure.

sedation in the ROSE trial, and sedation/analgesia and mechanical ventilation did not need to be optimized before randomization in contrast with the ACURASYS trial. Third, a high PEEP strategy was applied in the ROSE trial and prone positioning was not strongly encouraged.

Consequently, the proportion of patients in prone positioning was lower $(12.7 \%$ vs. $28.6 \%$ in the ACURASYS), and the use of corticosteroids was higher in the ROSE trial (25.2\%) than in the ACURASYS trial (19.2\%). The sedation protocol was followed differently in the ROSE trial, as the sedation level was slightly deeper than intended in the control group. In addition, more than $17 \%$ of the control group received an NMBA during the first 48 hours or during prone positioning sessions. Notably, 655 patients were not included before randomization because they had already received an NMBA. In addition, sedative and analgesia consumption was not reported in the ROSE trial, whereas the control and the interventional groups received the same doses in the ACURASYS trial. Lastly, patient-ventilator asynchrony was not measured in either trial.
However, although respiratory rates were approximately 2 breaths/min higher in the ROSE trial than those in the ACURASYS trial, adherence to the lung protective ventilation strategy in terms of tidal volume and driving pressure after the randomization seemed good in the former (Table 1); this strict use of low tidal volumes resulting in a low driving pressure might have reduced lung injury in the control group. The authors suggested that the higher PEEP levels used in the ROSE trial (i.e., $12.5-13.4$ vs. 9.6-9.7 $\mathrm{cmH}_{2} \mathrm{O}$ in the ACURASYS trial) may have blunted the potential treatment effects of NMBAs by improving lung recruitment and oxygenation quickly. However, compared to previous studies that have assessed higher vs. lower PEEP levels (6), the PEEP applied in the ROSE trial could be considered intermediate. Interestingly, recruitment maneuvers with high PEEP in patients with moderate to severe ARDS were associated with a higher mortality in a recent trial, compared to a low PEEP strategy with rather intermediate levels of PEEP; the hemodynamic effects and possible breath stackings with high PEEP are potential explanations of this finding (7). 
The intense central respiratory drive in patients with severe ARDS may preclude physicians from maintaining lung-protective ventilation without deep sedation and an NMBA, which can increase the risk for VILI $(8,9)$. NMBAs may alleviate this issue. Beyond improving oxygenation, NMBAs prevent active expiration, thus preventing expiratory atelectasis (10), stabilizing hemodynamics (11), and decreasing lung (and serum) inflammatory cytokines $(12,13)$. By contrast, spontaneous breathing (SB) with light sedation is associated with an increase in ventilator-free days and a shorter hospital stay $(14,15)$. However, despite these merits, SB can itself increase transpulmonary pressure in injured lungs and induce VILI (i.e., self-inflicted lung injury) $(16,17)$. Breath stacking dyssynchrony also generates higher tidal volumes than intended, potentially increasing lung injury risk in patients with ARDS $(18,19)$. Beitler et al. reported that breath stacking occurs in 27 breaths (7-59 breaths)/hour and is near completely eliminated during neuromuscular blockade, assuring provision of the intended lung-protective strategy (19). In addition, NMBAs are currently used during prone positioning, which was rarely performed in the ROSE trial. For instance, more than $90 \%$ of the patients included in the Proning Severe ARDS patients (PROSEVA) trial received NMBAs during prone positioning (20). Based on these data, minimizing SB during the early period of ARDS using deep sedation, and NMBAs (if necessary), seems reasonable.

The ROSE trial demonstrated that systematic use of NMBAs during the early phase of moderate to severe ARDS, before optimization of mechanical ventilation, sedation, and analgesia, does not reduce 90-day mortality. However, because patient-ventilator dyssynchrony, transpulmonary pressure, and inflammatory cytokines were not assessed in that trial, the physiological explanations of these results are still speculative. Based on the results of the ACURASYS and ROSE trials, NMBAs may only have a role when integrated in a bundle of mechanical ventilation care, including PEEP, sedation optimization, and prone positioning. We suggest that if lung-protective ventilation can be obtained with light sedation without patientventilator dyssynchrony, one should refrain from using NMBAs. By contrast, in cases of intense breath stacking, the clinician should maintain lung-protective ventilation to further prevent VILI, with the use of deep sedation and NMBAs if needed. In addition, the ROSE trial did not report the harmful effects of a short period of NMBAs on daily activity and quality of life at 12 months.

In conclusion, there is still room for the wise use of
NMBAs in the context of moderate to severe ARDS. With emergence of the concept of "ultra-lung protective ventilation" aimed at further reducing VILI with extracorporeal devices, NMBAs will likely continue to play a key role in patients with severe ARDS (21).

\section{Acknowledgments}

None.

\section{Footnote}

Conflicts of Interest: The authors have no conflicts of interest to declare.

Ethical Statement: The authors are accountable for all aspects of the work in ensuring that questions related to the accuracy or integrity of any part of the work are appropriately investigated and resolved.

\section{References}

1. Devlin JW, Skrobik Y, Gelinas C, et al. Clinical Practice Guidelines for the Prevention and Management of Pain, Agitation/Sedation, Delirium, Immobility, and Sleep Disruption in Adult Patients in the ICU. Crit Care Med 2018;46:e825-73.

2. Acute Respiratory Distress Syndrome Network, Brower RG, Matthay MA, Morris A, et al. Ventilation with lower tidal volumes as compared with traditional tidal volumes for acute lung injury and the acute respiratory distress syndrome. N Engl J Med 2000;342:1301-8.

3. Slutsky AS. Neuromuscular blocking agents in ARDS. N Engl J Med 2010;363:1176-80.

4. Papazian L, Forel JM, Gacouin A, et al. Neuromuscular blockers in early acute respiratory distress syndrome. $\mathrm{N}$ Engl J Med 2010;363:1107-16.

5. National Heart, Lung, and Blood Institute PETAL Clinical Trials Network, Moss M, Huang DT, et al. Early Neuromuscular Blockade in the Acute Respiratory Distress Syndrome. N Engl J Med 2019;380:1997-2008.

6. Briel M, Meade M, Mercat A, et al. Higher vs lower positive end-expiratory pressure in patients with acute lung injury and acute respiratory distress syndrome: systematic review and meta-analysis. JAMA 2010;303:865-73.

7. Writing Group for the Alveolar Recruitment for Acute Respiratory Distress Syndrome Trial I, Cavalcanti AB, 
Suzumura ÉA, et al. Effect of Lung Recruitment and Titrated Positive End-Expiratory Pressure (PEEP) vs Low PEEP on Mortality in Patients With Acute Respiratory Distress Syndrome: A Randomized Clinical Trial. JAMA 2017;318:1335-45.

8. Brochard L, Slutsky A, Pesenti A. Mechanical Ventilation to Minimize Progression of Lung Injury in Acute Respiratory Failure. Am J Respir Crit Care Med 2017;195:438-42.

9. Mauri T, Langer T, Zanella A, et al. Extremely high transpulmonary pressure in a spontaneously breathing patient with early severe ARDS on ECMO. Intensive Care Med 2016;42:2101-3.

10. Guervilly C, Bisbal M, Forel JM, et al. Effects of neuromuscular blockers on transpulmonary pressures in moderate to severe acute respiratory distress syndrome. Intensive Care Med 2017;43:408-18.

11. Putensen C, Mutz NJ, Putensen-Himmer G, et al. Spontaneous breathing during ventilatory support improves ventilation-perfusion distributions in patients with acute respiratory distress syndrome. Am J Respir Crit Care Med 1999;159:1241-8.

12. Forel JM, Roch A, Marin V, et al. Neuromuscular blocking agents decrease inflammatory response in patients presenting with acute respiratory distress syndrome. Crit Care Med 2006;34:2749-57.

13. Gainnier M, Roch A, Forel JM, et al. Effect of neuromuscular blocking agents on gas exchange in patients presenting with acute respiratory distress syndrome. Crit Care Med 2004;32:113-9.

Cite this article as: Park S, Schmidt M. Early neuromuscular blockade in moderate to severe acute respiratory distress syndrome: do not throw the baby out with the bathwater! J Thorac Dis 2019;11(11):E231-E234. doi: 10.21037/jtd.2019.10.25
14. Kress JP, Pohlman AS, O'Connor MF, et al. Daily interruption of sedative infusions in critically ill patients undergoing mechanical ventilation. $\mathrm{N}$ Engl J Med 2000;342:1471-7.

15. Zhou Y, Jin X, Lv Y, et al. Early application of airway pressure release ventilation may reduce the duration of mechanical ventilation in acute respiratory distress syndrome. Intensive Care Med 2017;43:1648-59.

16. Yoshida T, Nakahashi S, Nakamura MAM, et al. Volumecontrolled Ventilation Does Not Prevent Injurious Inflation during Spontaneous Effort. Am J Respir Crit Care Med 2017;196:590-601.

17. Brochard L. Ventilation-induced lung injury exists in spontaneously breathing patients with acute respiratory failure: Yes. Intensive Care Med 2017;43:250-2.

18. Akoumianaki E, Lyazidi A, Rey N, et al. Mechanical ventilation-induced reverse-triggered breaths: a frequently unrecognized form of neuromechanical coupling. Chest 2013;143:927-38.

19. Beitler JR, Sands SA, Loring SH, et al. Quantifying unintended exposure to high tidal volumes from breath stacking dyssynchrony in ARDS: the BREATHE criteria. Intensive Care Med 2016;42:1427-36.

20. Guérin C, Reignier J, Richard JC, et al. Prone positioning in severe acute respiratory distress syndrome. $\mathrm{N} \mathrm{Engl} \mathrm{J}$ Med 2013;368:2159-68.

21. Schmidt M, Pham T, Arcadipane A, et al. Mechanical Ventilation Management during ECMO for ARDS: An International Multicenter Prospective Cohort. Am J Respir Crit Care Med 2019;200:1002-12. 